\title{
POLYCYCLIC GROUP RINGS AND UNIQUE FACTORISATION RINGS
}

\author{
by KENNETH W. MacKENZIE
}

(Received 10 September, 1992)

\section{Noetherian unique factorisation rings.}

1.1. Introduction. The theory of unique factorisation in commutative rings has recently been extended to noncommutative Noetherian rings in several ways. Recall that an element $x$ of a ring $R$ is said to be normal if $x R=R x$. We will say that an element $p$ of a ring $R$ is (completely) prime if $p$ is a nonzero normal element of $R$ and $p R$ is a (completely) prime ideal. In [2], a Noetherian unique factorisation domain (or Noetherian $U F D$ ) is defined to be a Noetherian domain in which every nonzero prime ideal contains a completely prime element: this concept is generalised in [4], where a Noetherian unique factorisation ring (or Noetherian UFR) is defined as a prime Noetherian ring in which every nonzero prime ideal contains a nonzero prime element; note that it follows from the noncommutative version of the Principal Ideal Theorem that in a Noetherian UFR, if $p$ is a prime element then the height of the prime ideal $p R$ must be equal to 1 . Surprisingly many classes of noncommutative Noetherian rings are known to be UFDs or UFRs: see [2] and [4] for details. This theory has recently been extended still further, to cover certain classes of non-Noetherian rings: see [3].

It is shown in [2] that Noetherian UFDs have many good properties. In particular, let $R$ be a Noetherian UFD, and put

$$
\mathbf{C}=\mathbf{C}(R)=\left\{c \in R: c \in \mathscr{C}_{R}(p R) \text { for all prime elements } p \text { of } R\right\} .
$$

Then every nonzero element $r$ of $R$ can be written essentially uniquely in the form $r=c p_{1} \ldots p_{n}$ with $n \in \mathbb{Z}, c \in \mathbf{C}$ and $p_{1}, \ldots, p_{n}$ completely prime elements of $R$. Furthermore, $\mathrm{C}$ is an Ore subset of $R$ and in the localised ring $R_{\mathrm{C}}$ every one-sided ideal is two-sided and multiplication of ideals is commutative. Note that if $R$ is a commutative UFD then it follows from the Principal Ideal Theorem that the set $\mathbf{C}$ consists of units. In non-commutative rings this is not generally the case: in fact a result of M. P. Gilchrist and M. K. Smith states that if $R$ is a Noetherian UFD which is not commutative then every prime ideal of $R$ which has height two or more contains an element of $\mathbf{C}(R)$ [5]. It follows that in general the process of localising at the set $\mathbf{C}$ will bring about a considerable simplification in the structure of the ring involved.

The question naturally arises as to whether any of the above can be generalised to Noetherian UFRs, and in particular whether the set $\mathbf{C}$ consisting of those elements of a UFR $R$ which are regular modulo all of the height one prime ideals of $R$ is an Ore set. In general, this seems to be a very difficult problem, having certain resemblances to questions about localisability of cliques in Noetherian rings (however, see [3, Propositions 4.11 and 4.12]).

In the present paper we will investigate the problem above in a specific class of rings. K. A. Brown has given a complete characterisation of those polycyclic group rings which are UFDs and UFRs. We will use these results to prove that if $A$ is a commutative Noetherian UFD and $G$ is a polycyclic group such that the group ring $A G$ is a Noetherian UFR then the set $\mathbf{C}$ is indeed an Ore set in $A G$; we will also describe certain aspects of the ideal structure of the ring obtained by inverting the elements of $\mathbf{C}$. 
The research for this paper was carried out at the University of Edinburgh with the financial support of the Science and Engineering Research Council. I would like to thank Dr T. H. Lenagan, Dr A. W. Chatters and Prof. K. A. Brown for various useful discussions about the material dealt with in this paper.

1.2. Notation. If $R$ is a ring then $R^{\circ}$ will denote the set of units of $R$ and $\operatorname{Spec}^{l} R$ will denote the set of height-one prime ideals of $R$. If $R$ is a Noetherian UFR then we put

$$
\begin{gathered}
\mathbf{X}(R)=\{x \in R: x R=R x \neq 0\}, \\
\mathbf{X}_{1}(R)=\left\{p \in \mathbf{X}(R): p R \in \operatorname{Spec}^{1} R\right\}, \\
\mathbf{C}(R)=\mathscr{C}\left(\operatorname{Spec}^{1} R\right)=\cap\left\{\mathscr{C}_{R}(p R): p R \in \operatorname{Spec}^{1} R\right\} .
\end{gathered}
$$

We will abbreviate these to $\mathbf{X}, \mathbf{X}_{1}$ and $\mathbf{C}$ if the ring under consideration is clear from the context. Note that if $R$ is a Noetherian UFR then by definition we have $\operatorname{Spec}^{1} R=$ $\left\{p R: p \in \mathbf{X}_{1}(R)\right\}$.

1.3. The next result collects together various basic properties of UFRs that we will need.

Lemma. Let $R$ be a Noetherian UFR.

(i) If $0 \neq I \unlhd R$ then $I \cap \mathbf{X}(R) \neq \varnothing$.

(ii) If $P \in \operatorname{Spec} R$ and $x \in \mathbf{X}(R) \backslash P$ then $x \in \mathscr{C}_{R}(P)$.

(iii) $\mathbf{X}(R) \subseteq \mathscr{C}_{R}(0)$. $\mathscr{C}_{R}(0)$

(iv) If $p \in \mathbf{X}_{1}(R)$ then $\mathscr{C}_{R}(p R)=\mathscr{C}_{R}\left(p^{n} R\right)$ for all $n \geqslant 1$; furthermore, $\mathscr{C}_{R}(p R) \subseteq$

(v) $\mathbf{C}(R) \subseteq \mathscr{C}_{R}(0)$. $c^{\prime} \in \mathscr{C}(q R)$.

(vi) Suppose that $p, q \in X_{1}(R)$. If $c \in \mathscr{C}_{R}(q R)$ and $c^{\prime} \in R$ is such that $c p=p c^{\prime}$ then

(vii) If $p_{1}, \ldots, p_{n} \in \mathbf{X}_{1}(R)$ with $p_{i} R \neq p_{j} R$ for $i \neq j$ then $p_{1} R \cap \ldots \cap p_{n} R=$ $p_{1} \ldots p_{n} R$.

(viii) $\mathbf{X}(R)=\left\{u p_{1} \ldots p_{n}: u \in R^{\circ}, n \in \mathbb{N}\right.$ and $\left.p_{1}, \ldots, p_{n} \in \mathbf{X}_{1}(R)\right\}$.

(ix) If $x \in \mathbf{X}(R)$ then $\mathbf{C}(R) \subseteq \mathscr{C}_{R}(R x)$.

Proof. (i) Since $R$ is Noetherian, every nonzero ideal of $R$ contains a finite product of nonzero prime ideals of $R$; each of these prime ideals contains a prime element of $R$ (i.e. an element of $\mathbf{X}_{1}(R)$ ), and the product of these prime elements is clearly a nonzero normal element of $R$.

(ii) If $r \in R$ and $x r \in P$ then $x R r=R x r \subseteq P$. Since $P$ is a prime ideal and $x \notin P$ we have $r \in P$.

(iii) Apply (ii) with $P=0$.

(iv) See [3, Proposition 3.3].

(v) is immediate from (iv) and the definition of $\mathbf{C}(R)$.

(vi) Suppose that $r \in R$ and $c^{\prime} r \in q R$. If $p \notin q R$ then $c p r=p c^{\prime} r \in q R$ and so $p r \in q R$; but $p \in \mathscr{C}_{R}(q R)$ by (ii) and hence $r \in q R$. On the other hand, if $p \in q R$ then $p R=q R$ and so $c p r=p c^{\prime} r \in p q R=p^{2} R$, so $p r \in p^{2} R$ by (iv) and now $r \in p R=q R$, by (iii).

(vii) follows from [3, Lemma 3.1 and Theorem 3.3].

(viii) See [3, Lemma 3.4]. 
(ix) Suppose that $c \in \mathbf{C}(R), x \in \mathbf{X}(R)$ and $r \in R$ with $c r \in R x$. Part (viii) shows that $x=u p_{1} \ldots p_{n}$ for some $u \in R^{\circ}$ and some $p_{i} \in \mathbf{X}_{1}(R)$; so $c r \in R p_{1} \ldots p_{n} \subseteq R p_{n}$. Since $c \in \mathscr{C}_{R}\left(R p_{n}\right)$ we have $r \in R p_{n}$, say $r=r^{\prime} p_{n}$. Now $c r^{\prime} p_{n}=c r \in R p_{1} \ldots p_{n}$, and since $p_{n} \in \mathscr{C}_{R}(0)$ we have $c r^{\prime} \in R p_{1} \ldots p_{n-1}$. Induction on $n$ (the case $n=1$ being obvious) allows us to assume that $c \in \mathscr{C}_{R}\left(R p_{1} \ldots p_{n-1}\right)$, so that $r^{\prime} \in R p_{1} \ldots p_{n-1}$; but now $r=r^{\prime} p_{n} \in R p_{1} \ldots p_{n}=R x$ and it follows that $c \in \mathscr{C}_{R}(R x)$.

1.4. We now wish to give a sufficient condition for $\mathbf{C}(R)$ to be an Ore set in $R$.

Definition. Let $R$ be a Noetherian UFR. Put

$$
\begin{aligned}
\mathbf{D}(R) & =\left\{c p_{1} \ldots p_{n}: c \in \mathbf{C}(R), n \in \mathbb{N} \text { and } p_{j} \in \mathbf{X}_{1}(R)\right\} \\
& =\{c x: c \in \mathbf{C}(R) \text { and } x \in \mathbf{X}(R)\} .
\end{aligned}
$$

It follows from (vi) and (viii) of Lemma 1.3 that we also have

$$
\begin{aligned}
\mathbf{D}(R) & =\left\{p_{1} \ldots p_{n} c: c \in \mathbf{C}(R), n \in \mathbb{N} \text { and } p_{j} \in \mathbf{X}_{1}(R)\right\} \\
& =\{x c: c \in \mathbf{C}(R) \text { and } x \in \mathbf{X}(R)\} .
\end{aligned}
$$

We may view $\mathbf{D}(R)$ as being the set of elements of $R$ which do in fact have a unique factorisation as in a UFD. A UFR $R$ will be a UFD precisely when $\mathbf{D}(R)=R \backslash 0$.

1.5. The theorem below shows that the imposition of a certain Goldie-type condition on $\mathbf{D}(R)$ will ensure that $\mathbf{C}(R)$ is an Ore set. Recall $[\mathbf{8}, 6.4 .7]$ that a $\operatorname{ring} A$ is right bounded if every essential right ideal of $A$ contains a nonzero two-sided ideal. If $K$ is an essential right ideal of a ring $A$ then we will write $K \leqslant_{\mathrm{c}} A$.

THEOREM. Let $R$ be a Noetherian UFR. Then the following are equivalent:

(i) $\mathbf{C}(R)$ is a right Ore set in $R$ and $R_{\mathbf{C}(R)}$ is right bounded.

(ii) For all $c \in \mathscr{C}_{R}(0)$ there exists $r \in R$ such that $c r \in \mathbf{D}(R)$ (so $c r=d p_{1} \ldots p_{n}$ with $d \in \mathbf{C}(R)$ and $\left.p_{j} \in \mathbf{X}_{1}(R)\right)$.

(*) If $K$ is an essential right ideal of $R$ then $K \cap \mathbf{D}(R) \neq \varnothing$.

Proof. (ii) $\Leftrightarrow(*)$ is clear from Goldie's Theorem.

(i) $\Rightarrow(*)$. Suppose that (i) holds and denote the partial quotient ring of $R$ with respect to $\mathbf{C}$ by $R_{\mathbf{C}}$; it is clear that $R_{\mathbf{C}}$ is a UFR and $\operatorname{Spec}^{1} R_{\mathbf{C}}=\left\{p R_{\mathbf{C}}: p \in \mathbf{X}_{1}(R)\right\}$. Suppose that $K$ is an essential right ideal of $R$. Then $K R_{\mathbf{C}}$ is an essential right ideal of $R_{\mathbf{C}}$ and hence contains a nonzero two-sided ideal $I \unlhd R_{\mathbf{C}}$. Now $I \cap R$ is a nonzero two-sided ideal of $R$, so $I \cap \mathbf{X}(R) \neq \varnothing$ by $1.3(\mathrm{i})$; thus there exists $x \in \mathbf{X}(R)$ with $x \in I \subseteq K R_{\mathbf{C}}$. It follows that $x c \in K$ for some $c \in \mathbf{C}(R)$, so that (*) holds.

$(*) \Rightarrow$ (i). Suppose that (*) holds and let $c \in \mathbf{C}(R)$ and $r \in R$. Put $K=\{a \in R: r a \in$ $c R\} \leqslant R_{R}$. Since $\mathbf{C}(R) \subseteq \mathscr{C}_{R}(0)$ and $\mathscr{C}_{R}(0)$ is an Ore set by Goldie's Theorem, the right Ore condition shows that $K \cap \mathscr{C}_{R}(0) \neq \varnothing$, so by [8, 2.3.5], $K$ is essential in $R$. By (*) we know that $K$ meets $\mathbf{D}$, so there exist $d \in \boldsymbol{C}(R)$ and $x \in \mathbf{X}(R)$ with $d x \in K$. Thus $r d x \in c R$, say $c s=r d x \in R x$. By 1.3 (ix) $c \in \mathscr{C}(R x)$, so that $s \in R x$, say $s=t x$. But now $c t x=c s=r d x$, so $c t=r d$ with $d \in \mathbf{C}(R)$ and $t \in R$. Thus we have shown that $\mathbf{C}(R)$ is a right Ore set. By 1.3(v) we know that $\mathbf{C}(R)$ consists of regular elements. We may thus invert $\mathbf{C}$ with impunity; we will denote the localisation by $R_{\mathrm{C}}$.

Now suppose $F \leqslant_{\mathrm{e}} R_{\mathbf{C}}$. Then $F \cap R$ is an essential right ideal of $R$, so there exist $c \in \mathbf{C}(R)$ and $x \in \mathbf{X}(R)$ with $x c \in F \cap R \subseteq F$; but now $x=x c . c^{-1} \in F$ in $R_{\mathbf{C}}$, so that $F$ contains the nonzero two-sided ideal $x R_{\mathrm{C}}=R_{\mathrm{C}} x$. Thus $R_{\mathrm{C}}$ is bounded. 
Note. Of course, there is also a left-handed version of this theorem. Thus to prove that $\mathbf{C}(R)$ is a two-sided Ore set in some UFR $R$, we have to establish both condition (*) and its left-handed analogue. The rings that we will be dealing with are sufficiently symmetrical that having proved $(*)$, the proof of the left-handed version of $(*)$ will cause no difficulty.

1.6. We know of no UFR which fails to satisfy the condition $(*)$ of Theorem 1.5 , but we have been unable to prove that all UFRs satisfy (*). We now give some examples where $(*)$ can be shown to hold.

Proposition. Suppose that $R$ is a bounded UFR. Then $\mathbf{C}(R)$ consists of units and hence is an Ore set; thus $R$ is equal to $R_{\mathbf{C}}$ and so satisfies the conditions of Theorem 1.5.

Proof. Let $c \in \mathbf{C}(R) \subseteq \mathscr{C}(0)$. Then $c R$ contains some two-sided ideal of $R$, and hence some nonzero normal element $x$, so $x R \subseteq c R$, say $x=c r$. But $c r \in x R=R x$ and $c \in \mathscr{C}(x R)$, so $r \in R x$, say $r=s x$. We now have $x=c s x$ so $c s=1$ and $c \in R^{\circ}$.

1.7. Recall that if $S$ is a ring and $\Omega \subseteq \operatorname{Spec} S$ then $\Omega$ is said to satisfy the right intersection condition if, whenever $K \leqslant S_{S}$ with $K \cap \mathscr{C}(P) \neq \varnothing$ for all $P \in \Omega$, we have $K \cap \mathscr{C}(\Omega) \neq \varnothing$, where $\mathscr{C}(\Omega)=\cap\left\{\mathscr{C}_{R}(P): P \in \Omega\right\}$ (see [7, Chapter 7]).

Proposition. Suppose that $R$ is a UFR and $\operatorname{Spec}^{1} R$ satisfies the right intersection condition. Then $R$ satisfies $(*)$ of Theorem 1.5 .

Proof. Let $K \leqslant_{\mathrm{e}} R_{R}$ be maximal with respect to $K \cap \mathbf{D}=\varnothing$. If $p \in \mathbf{X}_{1}(R)$ put $K p^{-1}=\{r \in R: r p \in K\}$; note that $K p^{-1}$ is a right ideal of $R$ and that $K \leqslant K p^{-1}$. If $K<K p^{-1}$ then $K p^{-1} \cap \mathbf{D} \neq \varnothing$ and hence $K \cap \mathbf{D} \neq \varnothing$. Thus $K=K p^{-1}$ for all $p \in \mathbf{X}_{1}(R)$, so that

$$
r p \in K \Rightarrow r \in K
$$

Now let $P=p R \in \operatorname{Spec}^{1} R$, where $p \in \mathbf{X}_{1}(R) . P$ is localisable by $[4,2.2]$, and $R_{P}$ is a bounded local ring whose two-sided ideals are precisely $\left\{p^{n} R_{P}: n \in \mathbb{N}\right\}([6,1.3])$. Thus there exists $n$ with $p^{n} \in K R_{p}$, so $p^{n} c \in K$ for some $c \in \mathscr{C}_{R}(p R)$. Now there exists $c^{\prime} \in \mathscr{C}(p R)$ with $p^{n} c=c^{\prime} p^{n} \in K$; it follows from (1) that $c^{\prime} \in K$. Thus $K \cap \mathscr{C}(p R) \neq \varnothing$ for all $p \in \mathbf{X}_{1}(R)$, and the intersection condition on $\operatorname{Spec}^{1} R$ shows that $K \cap \mathbf{C}(R) \neq \varnothing$, so that $K \cap \mathbf{D}(R) \neq \varnothing$.

1.8. The last result above is not very revealing. It can be shown that if $R$ is a UFR then $\operatorname{Spec}^{1} R$ satisfies the intersection condition if $\left|\operatorname{Spec}^{1} R\right|<\infty$ or if $R$ is an algebra over a field $k$ with $\left|\operatorname{Spec}^{1} R\right|<|k|$ (see [7, 7.2.12]). However, it follows easily from the Principal Ideal Theorem that if $R$ is a commutative UFR then $\operatorname{Spec}^{1} R$ will satisfy the intersection condition precisely when $R$ has Krull dimension 1. Thus it seems that examples of UFRs whose height one primes satisfy the intersection condition will be rare.

Despite these remarks, the condition $(*)$ of Theorem 1.5 is useful. We will show that if $A$ is a commutative UFD and $G$ is a polycyclic-by-finite group such that the group ring $A G$ is a UFR then $A G$ satisfies (*). Then basic idea is to show that in this situation $G$ has a normal subgroup $H$ of finite index such that $A H$ is a UFD; there is then a sufficiently close connection between $\operatorname{Spec} A G$ and $\operatorname{Spec} A H$ to allow us to prove (*). This will be done in the next section. 


\section{Group rings and Noetherian UFRs.}

2.1. It is a well-known result of $\mathrm{P}$. Hall that if $A$ is a commutative Noetherian ring and $G$ is a polycyclic-by-finite group then $A G$, the group ring of $G$ over $A$, is a Noetherian ring (see $[9,10.2 .8]$ or $[8,1.5 .12]$ ). When $A$ is a commutative UFD, necessary and sufficient conditions on $G$ for $A G$ to be a UFD or UFR are given in [1] and [1']. Using these we will prove the following theorem.

THEOREM. Let $A$ be a commutative Noetherian UFD and $G$ a polycyclic-by-finite group. If $A G$ is a UFR then $G$ has a normal (in fact, characteristic) subgroup $H$ of finite index such that $A H$ is a UFD.

The proof is group-theoretic and is deferred until Section 3.

2.2. In the situation of Theorem 2.1 there is a strong relationship between $\operatorname{Spec} A G$ and Spec $A H$, which we will now sketch (see [10, Chapters 14 and 16] for full details).

Here and in 2.3 we suppose that $A$ is a ring, $G$ is a group, and $H$ is a normal subgroup of finite index in $G$. Put $S=A H$ and $R=A G$. We can identify $R$ with a crossed product $S * \bar{G}$, where $\bar{G}$ is the finite group $G / H$ (cf. [10, Chapter 1]).

Firstly, note that $G$ acts naturally on the lattice of (two-sided) ideals of $A H$, via

$$
I \mapsto I^{g}=\left\{g^{-1} x g: x \in I\right\} \quad(I \unlhd R) .
$$

An ideal $I$ of $S$ is $G$-stable if $I^{g} \subseteq I$ for all $g \in G$ (we write $I \unlhd_{G} S$ if this is the case). A proper ideal $Q$ of $S$ is $G$-prime if, whenever $I$ and $J$ are $G$-stable ideals of $S$ with $I J \subseteq Q$, then $I \subseteq Q$ or $J \subseteq Q$; we denote the set of. $G$-prime ideals of $S$ by $\operatorname{Spec}^{G} S$. Note that every ideal of $S$ is $H$-stable, so that $\vec{G}$ acts on $S$. If $I$ is an ideal of $S$ then it is not hard to see that $\left\{I^{g}: g \in G\right\}=\left\{I^{\bar{g}}: \bar{g} \in \bar{G}\right\}$; it follows from this that every ideal of $S$ has only finitely many (in fact, at most $[G: H]$ ) conjugates under the action of $G$. It is clear that the $\bar{G}$-stable ideals of $S$ coincide with the $G$-stable ideals, so that $\operatorname{Spec}^{\bar{G}} S=\operatorname{Spec}^{C} S$. The $G$-prime ideals of $S$ can be described explicitly (see [10, Lemma 14.2]). Firstly, if $I$ is an ideal of $S$, write

$$
I^{G}=\bigcap\left\{I^{g}: g \in G\right\} .
$$

It is easily seen that $I^{G}$ is a $G$-stable ideal of $S$ for every ideal $I$ of $S$, and that $P^{G}$ is a $G$-prime ideal for every prime ideal $P$. In fact, if $Q$ is any $G$-prime of $S$ and $P$ is any prime ideal of $S$ which is minimal over $Q$ then $Q=P^{G}=P^{\bar{G}}$; it follows that there exist $g_{1}, g_{2}, \ldots, g_{1} \in G$ such that

$$
Q=P^{g_{1}} \cap P^{g_{2}} \cap \ldots \cap P^{g_{t}}
$$

Let $\left\{t_{1}, \ldots, t_{n}\right\}$ be a right transversal to $H$ in $G$; we may assume that $t_{1}=1$, and we have

$$
R=S \oplus S t_{2} \oplus \ldots \oplus S t_{n},
$$

a finite normalising extension (see $[10$, p. 159] or $[8, \S \$ 10.1,10.2]$ ). We may regard $S$ as a subring of $R$, and this gives us a means of passing from ideals of $R$ to ideals of $S$ and back. If $P \in \operatorname{Spec} R$ then it turns out that $P \cap S \in \operatorname{Spec}^{G} S$ ([10, Lemma 14.1]), so that $P \cap S=Q^{G}$ for some $Q \in \operatorname{Spec} S$. Moreover, if $I$ is a $G$-stable ideal of $S$ then $I R=R I$ is an ideal of $R$ : in particular, if $Q \in \operatorname{Spec} S$ then $Q^{G} R$ is an ideal of $R$. It can be shown that a 
prime ideal $P$ of $R$ is minimal over $Q^{G} R$ if and only if $P \cap S=Q^{G}$ ([10, Theorem 16.2]), and thus if and only if $P$ lies over $Q$ (see $[10,16.6(\mathrm{i})]$ ).

We thus have a means of passing between $\operatorname{Spec} S$ and $\operatorname{Spec} R$. It is shown in $[10,16.6$, 16.8] that this process satisfies analogues of the Going Up, Going Down and Incomparability properties of commutative algebra; also, height is preserved by this process.

2.3. We also need a result on Ore sets in group rings.

Lemma. If $\mathscr{S}$ is a (right) Ore set in $S$ and $\mathscr{P}^{g} \subseteq \mathscr{S}$ for all $g \in G$ then $\mathscr{S}$ is a (right) Ore set in $R$.

Proof. The proof of [11, Lemma 2.6] can be used without changes.

2.4. We can now return to UFRs. We will use the following notation for the remainder of Section 2: $A$ is a commutative Noetherian UFD, $G$ is a polycyclic-by-finite group such that $A G$ is a UFR, $H$ is a normal subgroup of $G$ with $[G: H]<\infty$ such that $A H$ is a UFD, as given by Theorem 2.1 , and $R=A G$ and $S=A H$ are the group rings of $G$ and $H$ over $A$.

2.5. Lemma. $\mathbf{C}(S)=\mathbf{C}(R) \cap S$.

Proof. $\mathbf{C}(S)$ is an Ore set in $S$ by $[2,2.5]$, and it is easily seen to be $G$-invariant (essentially because $\operatorname{Spec}^{1} S$ is $G$-stable); Lemma 2.3 now shows that $\mathbf{C}(S)$ is an Ore set in $R$.

(i) $\mathbf{C}(S) \subseteq \mathbf{C}(R)$. Let $P \in \operatorname{Spec}^{1} R$ and put

$$
K=\{r \in R: c r \in P \text { for some } c \in \mathbf{C}(S)\} \leqslant R_{R} .
$$

Since $\mathbf{C}(S)$ is an Ore set in $R, K$ is in fact a two-sided ideal of $R$ (cf. [8, 2.1.9]). Now $P \subseteq K$. Suppose that $K>P$ : then Goldie's Theorem shows that $K \cap \mathscr{C}_{R}(P) \neq \varnothing$, and hence $c d \in P$ for some $c \in \mathbf{C}(S)$ and some $d \in \mathscr{C}_{R}(P)$. Thus $c \in P \cap \mathbf{C}(P)$. Let $\mathfrak{p} \in \operatorname{Spec} S$ be minimal over $P \cap S$; it follows from $[10,6.8]$ that $\mathfrak{p}$ has height one in $S$. Since $c \in \mathbf{C}(S)$ we have $c \in \mathscr{C}_{S}(\mathfrak{p})$; but we also have $c \in P \cap S \subseteq \mathfrak{p}$, a contradiction.

Thus $K=P$, and hence $\mathbf{C}(S) \subseteq \mathscr{C}_{R}(P)$. Since $P$ was an arbitrary height one prime of $R$ we have $\mathbf{C}(S) \subseteq \mathbf{C}(R)$.

(ii) $\mathbf{C}(R) \cap S \subseteq \mathbf{C}(S)$. Let $c \in \mathbf{C}(R) \cap S$ and let $\mathfrak{p} \in \operatorname{Spec}^{1} S$. Recall from 2.2 that $\mathfrak{p}$ has only finitely many distinct $G$-conjugates and denote them by $\mathfrak{p}_{1}=\mathfrak{p}, \mathfrak{p}_{2}, \ldots, \mathfrak{p}_{n}$. Let $s \in S$ with $c s \in \mathfrak{p}$. Then

$$
c s \mathfrak{p}_{2} \ldots \mathfrak{p}_{n} \subseteq \mathfrak{p}_{1} \mathfrak{p}_{2} \ldots \mathfrak{p}_{n} \subset \mathfrak{p}_{1} \cap \ldots \cap \mathfrak{p}_{n}=\mathfrak{p}^{G}
$$

Let $P \in \operatorname{Spec} R$ be minimal over $\mathfrak{p}^{G} R$. By $[10,6.8]$ we have $P \in \operatorname{Spec}^{1} R$ (so that $\left.c \in \mathscr{C}_{R}(P)\right)$, and $[10,16.6$, (i) and (iii) $]$ show that $P \cap S=\mathfrak{p}^{G}$. Thus

$$
\operatorname{cs} \mathfrak{p}_{2} \ldots \mathfrak{p}_{n} \subseteq \mathfrak{p}^{G}=P \cap S
$$

and since $c \in \mathscr{C}(P)$ we have $s \mathfrak{p}_{2} \ldots \mathfrak{p}_{n} \subseteq P \cap S=\mathfrak{p}^{G} \subseteq \mathfrak{p}$. Since $\mathfrak{p}$ is prime and $\mathfrak{p}_{j} \ddagger \mathfrak{p}$ for $j \neq 1$ we see that $s \in \mathfrak{p}$. Thus $c \in \mathscr{C}_{S}(\mathfrak{p})$ for all $\mathfrak{p} \in \operatorname{Spec}^{1} S$, which is to say that $c \in \mathbf{C}(S)$.

2.6. Lemma. If $K$ is an essential right ideal of $R$ then $K \cap S$ is an essential right ideal of $S:$ in particular it is nonzero. 
Proof. Recall from 2.2 that $R$ is a finitely generated module over the Noetherian ring $S$, so that $R_{S}$ is Noetherian. Suppose that $L \leqslant S_{S}$ with $(K \cap S) \cap L=0$. Since $K$ is essential in the prime ring $R$ it contains a regular element $c \in \mathscr{C}_{R}(0)$, and we have $c R \cap L \subseteq$ $K \cap L=0$. A standard argument now shows that there is an infinite direct sum

$$
L \oplus c L \oplus c^{2} L \oplus \ldots
$$

of $S$-submodules of $R$. If $L \neq 0$ this contradicts the fact that $R_{S}$ is Noetherian.

2.7. Lemma. If $x \in \mathbf{X}(S)$ then there exists $\bar{x} \in S$ such that $x \bar{x} \in \mathbf{X}(R)$.

Proof. Suppose firstly that $p \in \mathbf{X}_{1}(S)$, so that $p S \in \operatorname{Spec}^{l} S$. Now $p S$ has only finitely many distinct $G$-conjugates, and these are all height one primes of $S$ and thus principal: suppose that there are $m$ of them and choose normal generators $p_{1}=p, p_{2}, \ldots, p_{m}$. Now by Lemma $1.3(\mathrm{vii}),(p S)^{G}=p_{1} S \cap p_{2} S \cap \ldots \cap p_{m} S=p_{1} p_{2} \ldots p_{m} S$. Put $\bar{p}=p_{2} \ldots p_{m} \epsilon$ $\mathbf{X}(S)$, so that $(p S)^{G}=p \bar{p} S=S p \bar{p}$. We have

$$
p \bar{p} R=(p \bar{p} S) R=(p S)^{G} R=R(p S)^{G}=R(S p \bar{p})=R p \bar{p},
$$

so that $p \bar{p} \in \mathbf{X}(R)$. Now suppose $x$ is any element of $\mathbf{X}(S)$. We know by 1.3(viii) that $x$ can be written in the form $x=u q_{1} q_{2} \ldots q_{n}$ with $u \in S^{\circ} \subseteq R^{\circ}$ and $q_{j} \in \mathbf{X}_{1}(S)$. Put $y=u q_{1} \ldots q_{n-1}$ and $q=q_{n}$, so that $x=y q$ with $y \in \mathbf{X}(S)$ and $q \in \mathbf{X}_{1}(S)$. Using induction on $n$ (the case $n=0$ being obvious) we may assume that there exists $\bar{y} \in S$ with $y \bar{y} \in \mathbf{X}(R)$. Since $q$ is a normal element of $S$ there exists $y^{\prime} \in S$ with $q y^{\prime}=\bar{y} q$. Put $\bar{x}=y^{\prime} \bar{q}$, where $\bar{q}$ is defined as in (*). Then $x \bar{x}=y q y^{\prime} \bar{q}=y \bar{y} q \bar{q}$. We know that $y \bar{y}$ and $q \bar{q}$ both lie in $\mathbf{X}(R)$, so $x \bar{x} \in \mathbf{X}(R)$ also.

2.8. Recall that $\mathbf{D}(R)=\{c x: c \in \mathbf{C}(R)$ and $x \in \mathbf{X}(R)\}$.

Theorem. Let $K \leqslant_{\mathrm{c}} R_{R}$. Then $K \cap \mathbf{D}(R) \neq \varnothing$.

Proof. By Lemma 2.6 we have $K \cap S \neq 0$. Since $S$ is a UFD there exist $c \in \mathbf{C}(S)$ and $p_{1}, \ldots, p_{n} \in \mathbf{X}_{1}(S)$ with $c p_{1} \ldots p_{n} \in K \cap S$ (see $[2,2.1]$ ). Put $x=p_{1} \ldots p_{n} \in \mathbf{X}(S)$. By Lemma 2.7 there exists $\bar{x} \in S$ with $x \bar{x} \in \mathbf{X}(R)$; but since $\mathbf{C}(S) \subseteq \mathbf{C}(R)$ (Lemma 2.5), we have $c x \bar{x} \in K \cap \mathbf{D}(R)$.

2.9. Corollary. Let $A$ be a commutative Noetherian UFD and $G$ a polycyclic-byfinite group such that the group ring $R=A G$ is a Noetherian UFR.

(i) $\mathbf{C}=\mathbf{C}(R)$ is an Ore set in $R$ and $R_{\mathbf{C}}$ is bounded.

(ii) Either $R$ satisfies a polynomial identity and $R=R_{\mathrm{C}}$ is a bounded UFR or $R_{\mathrm{C}}$ has classical Krull dimension one and all of its two-sided ideals are principal.

Proof. (i) follows from Theorems 2.8 and 1.5, and their left-handed analogues. Note that $\mathbf{C}(S)$ is an Ore set in $R$ by $[2,2.5]$ and Lemma 2.3. If $c \in \mathbf{C}(R) \subseteq \mathscr{C}_{R}(0)$ then the proof of Theorem 2.8 shows that there exists $r \in R$ such that $c r=d x$ for some $d \in \mathbf{C}(S)$ and $x \in \mathbf{X}(R)$; now $c \in \mathscr{C}_{R}(x R)$ (by Lemma 1.3(ix)) so that $r=r^{\prime} x$, say. But now we have $c r^{\prime}=d \in \mathbf{C}(S)$. It follows that $R_{\mathrm{C}(R)}=R_{\mathbf{C}(S)}$.

(ii) If $H$ is abelian then $S=A H$ is a commutative ring and since $R=A G$ is a finite extension of $A H,[8,13.1 .13$ (iii)] shows that $R$ is a PI-ring. It follows from [8, 13.6.6] that in this case $R$ is a bounded ring and so by Proposition 1.6, $\mathrm{C}(R)=R^{\circ}$.

Thus if $R$ is not a PI-ring, $H$ cannot be abelian and hence $S$ cannot be commutative. In this case it follows from [5] that every prime of $S$ of height 2 or more meets $\mathbf{C}(S)$ and 
hence $\mathbf{C}(R)$. Suppose $P \in \operatorname{Spec} R$ with ht ${ }_{R}(P) \geqslant 2$ and let $p \in \operatorname{Spec} S$ with $P \cap S=\mathfrak{p}^{G}$. Then ht $\mathrm{ht}_{S} \mathfrak{p}=\mathrm{ht}_{R} P \geqslant 2([\mathbf{1 0}, 16.8])$ so that $\mathfrak{p} \cap \mathbf{C}(S) \neq \varnothing$. Now $\mathfrak{p}^{G}$ is the product of a finite number of $G$-conjugates of $\mathfrak{p}$, each of which must also meet $\mathbf{C}(S)$, since $\mathbf{C}(S)$ is $G$-stable. Thus $\mathfrak{p}^{G} \cap \mathbf{C}(S) \neq \varnothing$, so that $P \cap \mathbf{C}(S) \neq \varnothing$ and hence $P \cap \mathbf{C}(R) \neq \varnothing$, so that $P R_{\mathbf{C}(R)}=$ $R_{\mathrm{C}(R)}$. Thus all maximal ideals of $R_{\mathrm{C}}$ have height one and so $R_{\mathrm{C}}$ has classical Krull dimension 1. Since all of the maximal ideals of $R_{\mathrm{C}}$ are principal, and hence invertible, $R_{\mathrm{C}}$ is an Asano order (see [8, 5.2.6]); in an Asano order multiplication of ideals is commutative and every two-sided ideal is a unique product of maximal ideals $([\mathbf{8}, \S 5.2])$. Thus every two-sided ideal of $R_{\mathrm{C}}$ is a product of principal ideals, and so is principal.

\section{Proof of Theorem 2.1.}

3.1. If $A$ is a commutative Noetherian UFD and $G$ is a polycyclic-by-finite group then K. A. Brown ([1] and $\left.\left[\mathbf{1}^{\prime}\right]\right)$ has given necessary and sufficient conditions for the group ring $A G$ to be a Noetherian UFD or UFR: we will use these results to prove Theorem 2.1. We must recall some notation and definitions before we can state these results.

Definition. Let $G$ be a group. We denote the centre of $G$ by $Z(G)$. If $S$ is a subset of $G$ then the centraliser of $S$ (in $G$ ) is $\mathbf{C}_{G}(S)=\left\{x \in G: s^{x}=s \forall s \in S\right\}$, and the normaliser of $S$ (in $G$ ) is $\mathbf{N}_{G}(S)=\left\{x \in G: S^{x} \subseteq S\right\}$. The f.c. (finite conjugate) subgroup of $G$ is $\Delta(G)=\left\{x \in G:\left[G: \mathbf{C}_{G}(x)\right]<\infty\right\}=\left\{x \in G:\left|\left\{x^{g}: g \in G\right\}\right|<\infty\right\}$, and $\Delta^{+}(G)=\{x \in \Delta(G):$ $o(x)<\infty\}$, where $o(x)=\inf \left\{k>0: x^{k}=1\right\}$ is the order of $x \in G$. A subset $S$ of $G$ is orbital if $\left|\left\{S^{g}: g \in G\right\}\right|<\infty$. It is not hard to show that the obvious map between $\left\{S^{g}: g \in G\right\}$ and $\left\{g \mathbf{N}_{G}(S): g \in G\right\}$ is a bijection, so that $S$ is orbital if and only if $\left[G: \mathbf{N}_{G}(S)\right]<\infty$.

Now suppose that $G$ is a polycyclic-by-finite group. A plinth of $G$ is a torsionfree abelian orbital subgroup $B \leqslant G$ such that $B \otimes_{\mathbb{Z}} \mathbb{Q}$ is an irreducible $\mathbb{Q} T$-module for all subgroups $T$ of $\mathbf{N}_{G}(B)$ with $\left[\mathbf{N}_{G}(B): T\right]<\infty$. A plinth $B$ is centric if $\left[G: \mathbf{C}_{G}(B)\right]<\infty$, otherwise is is eccentric. The group $G$ is dihedral free if it contains no orbital subgroup isomorphic to the infinite dihedral group $D=\left\langle a, b: a^{2}=1, b^{a}=b^{-1}\right\rangle$.

In 3.2 and 3.3 we assume that $A$ is a commutative Noetherian ring and $G$ is a polycyclic-by-finite group.

3.2. TheOREM ([1, Theorem E], $\left[\mathbf{1}^{\prime}\right.$, Theorem $\left.\left.\mathrm{E}^{\prime}\right]\right)$. If $A$ is a UFD then $A G$ is a UFD if and only if (i) $G$ is torsionfree, (ii) $G / \Delta(G)$ is torsionfree, and (iii) all plinths of $G$ are central (i.e. contained in $Z(G)$ ).

3.3. TheOREM ([1, Theorem D]). If $A$ is a UFD then $A G$ is a UFR if and only if (i) $\Delta^{+}(G)=1$, (ii) $G$ is dihedral free, and (iii) every plinth of $G$ is centric.

3.4. Now suppose that $G$ is a polycyclic-by-finite group such that $A G$ is a UFR: to prove Theorem 2.1 we must find a normal subgroup $H$ of finite index in $G$ such that $H$ satisfies the conditions (i), (ii) and (iii) of Theorem 3.2. The next few results will prepare us for this.

Lemma. Let $G$ be a group. If $H \leqslant G$ and $[G: H]<\infty$ then $\Delta(H)=\Delta(G) \cap H$. In particular, if $\Delta(G) \leqslant H \leqslant G$ then $\Delta(H)=\Delta(G)$. 
Proof. It is clear that $\Delta(G) \cap H \subseteq \Delta(H)$, for if an element $x \in H$ has only finitely many $G$-conjugates then it certainly has only finitely many $H$-conjugates. Conversely, if $x \in \Delta(H)$ then $\left[H: \mathbf{C}_{H}(x)\right]<\infty$, so that $\left[G: \mathbf{C}_{H}(x)\right]<\infty$. Hence $\left[G: \mathbf{C}_{G}(x)\right]<\infty$, so $x \in \Delta(G)$.

3.5. Proposition. Let $G$ be a polycyclic-by-finite group. If $H$ is a normal subgroup of finite index in $G$ then every plinth of $H$ is also a plinth of $G$.

Proof. This follows easily from the definition.

3.6. Proposition. Let $G$ be a polycyclic-by-finite group. Then $G$ has a characteristic poly-(infinite cyclic) subgroup $E=E(G)$ of finite index such that $E / \Delta(E)$ is poly-(infinite cyclic) and $Z(E)=\Delta(E)=\Delta(G) \cap E$.

Proof. It follows from $[9,10.2 .5]$ that $G$ has a characteristic poly-(infinite cyclic) subgroup $F$ of finite index in $G$. The subgroup $F$ is torsionfree by $[9,10.2 .4]$ and hence $\Delta^{+}(F)=1:[9,4.1 .6]$ now shows that $\Delta(F)$ is a characteristic torsionfree abelian subgroup of $F$, and hence of $G$. Also, $\Delta(F)$ is poly-(infinite cyclic) $([9,10.2 .4])$ and hence is finitely generated, say $\Delta(F)=\left\langle a_{1}, \ldots, a_{n}\right\rangle$. Let $K=\mathbf{C}_{F}(\Delta(F))$, which is characteristic in $G$ since $F$ and $\Delta(F)$ are, and has finite index in $G$, since $[G: F]<\infty, K=\bigcap\left\{\mathbf{C}_{F}\left(a_{i}\right): 1 \leqslant i \leqslant n\right\}$. Now $\Delta(F) \subseteq K$, so by Lemma $3.4 \quad \Delta(F)=\Delta(K) \supseteq Z(K)$. By the definition of $K, \Delta(F) \subseteq Z(K)$, so

$$
Z(K)=\Delta(K)=\Delta(F)
$$

We have $K \leqslant F$ so that $K$ is poly-(infinite cyclic) $[9,10.2 .4]$ and $K / \Delta(K)$ is polycyclic-byfinite. Applying $[9,10.2 .5]$ to $K / \Delta(K)$ we obtain a subgroup $E=E(G)$ which is characteristic in $K$ with $\Delta(K) \subseteq E, E / \Delta(K)$ poly-(infinite cyclic) and $[K / \Delta(K): E / \Delta(K)]=$ $[K: E]<\infty$. By Lemma 3.4 again, $\Delta(E)=\Delta(K)=\Delta(F)$. We have $\Delta(E)=\Delta(K)=$ $\Delta(K) \cap E=Z(K) \cap E \subseteq Z(E) \subseteq \Delta(E)$, so

$$
\Delta(E)=Z(E)=\Delta(K)=Z(K)=\Delta(F)=\Delta(G) \cap E .
$$

Now $E$ is poly-(infinite cyclic) (since it is a subgroup of $K$ ), is of finite index in $G$, and is characteristic in $G$, as required.

\subsection{The proof of Theorem 2.1.}

THEOREM. Let $A$ be a commutative Noetherian UFD and $G$ a polycyclic-by-finite group such that the group ring $A G$ is a UFR. If we put $H=E(G)$ then $H \unlhd G,[G: H]<\infty$ and $A H$ is a UFD.

Proof. Let $H=E(G)$ and recall from Proposition 3.6 that $H$ is a normal subgroup of finite index in $G$ such that $Z(H)=\Delta(H)=\Delta(G) \cap H$. We know that both $H$ and $H / \Delta(H)$ are poly-(infinite cyclic) and hence torsionfree. Thus by Theorem 3.2 it suffices to show that all plinths of $H$ are central.

Let $B \leqslant H$ be a plinth of $H$; by Proposition 3.5, $B$ is also a plinth of $G$, and since $A G$ is a UFR, $B$ must be centric in $G$, i.e. $\left[G: \mathbf{C}_{G}(B)\right]<\infty$. Now let $b \in B$; then $\left[H: \mathbf{C}_{H}(b)\right] \leqslant\left[H: \mathbf{C}_{H}(B)\right]=\left[H: \mathbf{C}_{G}(B) \cap H\right] \leqslant\left[G: \mathbf{C}_{G}(B) \cap H\right] \leqslant\left[G: \mathbf{C}_{G}(B)\right][G: H]<\infty$. Thus $b \in \Delta(H)=Z(H)$, so $B \subseteq Z(H)$ is central in $H$. 


\section{REFERENCES}

1. K. A. Brown, Height one primes of polycyclic group rings, J. London Math. Soc. (2) 32 (1985), 426-438.

1'. K. A. Brown, Corrigendum and addendum, Height one primes of polycyclic group rings, J. London Math. Soc. (2) 38 (1988), 421-22.

2. A. W. Chatters, Noncommutative unique factorisation domains, Math. Proc. Cambridge Philos. Soc. 95 (1984), 49-54.

3. A. W. Chatters, M. P. Gilchrist and D. Wilson, Unique factorisation rings, Proc. Edinburgh Math. Soc. (2) 35 (1992), 255-269.

4. A. W. Chatters and D. A. Jordan, Noncommutative unique factorisation rings, J. London Math. Soc. (2) 33 (1986), 22-32.

5. M. P. Gilchrist and M. K. Smith, Noncommutative UFDs are often PIDs, Math. Proc. Cambridge Philos. Soc. 95 (1984), 417-419. $441-449$

6. C. R. Hajarnavis and T. H. Lenagan, Localisation in Asano orders, J. Algebra 21 (1972),

7. A. V. Jategaonkar, Localization in Noetherian rings, London Math. Soc. Lecture Note Series 98 (Cambridge University Press, 1986). 1987).

8. J. C. McConnell and J. C. Robson, Noncommutative Noetherian rings (Wiley-Interscience, 1985)

9. D. S. Passman, The algebraic structure of group rings (Wiley-Interscience, 1977), (Krieger,

10. D. S. Passman, Infinite crossed products (Academic Press, 1989).

11. P. F. Smith, Quotient rings of group rings, J. London Math. Soc. (2) 3 (1971), 645-660.

DepartMENT OF MATHEMATiCS

UNIVERSITY OF EDINBURGH

James Clerk Maxwell Building

KING'S BUILDINGS

MAYFIELD ROAD

EDINBURGH EH9 3JZ
Present address:

Department of MaTHEMATiCS

UNIVERSITY OF LIVERPOOL

P.O. Box 147

LiverPool L69 3BX 